\title{
Degree Relatives are Ordinary Relatives
}

\author{
Alastair Butler \\ University of York
}

\section{Introduction}

This paper aims to bring us nearer to a unified semantics for all relative clauses. The focus is on providing a single analysis for ordinary restrictives and degree relatives that avoids recourse to degrees. Before turning to the properties that single out degree relatives it will be helpful to first note that apparently ordinary restrictives can (at least in principle) receive two kinds of readings: restrictive and exhaustive.

\subsection{Restrictive vs. exhaustive readings of relatives}

Carlson (1977) notes that (1), which has the appearance of an ordinary restrictive relative, is ambiguous. It has its expected restrictive reading and what I will call an exhaustive reading (the formulation of which is due to Zeevat 1994, see also Butler 2001). ${ }^{1}$

(1) Peter ate everything that would fit in his pocket.

Restrictive reading:

Peter ate everything (relevant) that was of an appropriately small size.

$\forall x(P(x) \rightarrow A(x))$. (Using $P$ for would fit in Peter's pocket and $A$ for Peter ate.)

Exhaustive reading:

Peter ate a pocketfull of something. ${ }^{2}$

$\exists x(P(x) \wedge \neg \exists y(x \neq y \wedge P(y) \wedge \square(P(y) \rightarrow P(x))) \wedge A(x))$.

Presence of a $w h$-relativiser, like which in (2a), or a determiner that is not definite or universal, like some in $(2 \mathrm{~b})$, gives a restrictive reading only.

(2) a. Peter ate everything which would fit in his pocket.

b. Peter ate something that would fit in his pocket.

Importantly, (1) shows that restrictive and exhaustive readings can be true under different conditions. Suppose the domain of quantification is restricted to food in Peter's kitchen. To be true, the restrictive reading needs Peter to eat all the food small enough to fit in his pocket. In contrast, the exhaustive reading can still be true if an apple is left uneaten on the worktop. Rather it requires Peter to eat as much food as he is able to cram in his pocket. This difference arises with (1) because of the role of size in calculating values that would fit in Peter's pocket $(P)$. However, it is more often the case that the truth conditions of the two readings coincide, as in (3)-which perhaps explains why the distinction is often overlooked. 
(3) Mary collected every book that Peter read.

Restrictive reading:

$\forall x(R(x) \rightarrow M(x))$. (Using $R$ for Peter read _ book(s) and $M$ for Mary collected.)

Exhaustive reading:

Mary collected some books such that Peter read them and there wasn't an alternative collection of books that Peter read that contained more books. ${ }^{3}$

$\exists x(R(x) \wedge \neg \exists y(x \neq y \wedge R(y) \wedge \square(R(y) \rightarrow R(x))) \wedge M(x))$.

Occasionally the restrictive reading is not available, leaving only the exhaustive reading. For example, (4) and (5) contain relatives with a descriptive content that fails to return unique values.

(4) The money that Peter can live on is small.

Restrictive reading:

The unique money that Peter can live on is small!

$\exists x\left(M_{1}(x) \wedge \forall y\left(M_{1}(y) \rightarrow x=y\right) \wedge S(x)\right)$. (Using $M_{1}$ for Peter can live on _ money and $S$ for is small.)

Exhaustive reading:

Some money that is the minimum Peter can live on is small. ${ }^{4}$

$\exists x\left(M_{1}(x) \wedge \neg \exists y\left(x \neq y \wedge M_{1}(y) \wedge \square\left(M_{1}(y) \rightarrow M_{1}(x)\right)\right) \wedge S(x)\right)$.

(5) The money that Peter can spend is small.

Restrictive reading:

The unique money that Peter can spend is small!

$\exists x\left(M_{2}(x) \wedge \forall y\left(M_{2}(y) \rightarrow x=y\right) \wedge S(x)\right)$. (Using $M_{2}$ for Peter can spend__ money.)

Exhaustive reading:

Some money that is the maximum Peter can spend is small..$^{5}$

$\exists x\left(M_{2}(x) \wedge \neg \exists y\left(x \neq y \wedge M_{2}(y) \wedge \square\left(M_{2}(y) \rightarrow M_{2}(x)\right)\right) \wedge S(x)\right)$.

To sum up this detour, ordinary restrictive relatives can in principle support a restrictive reading and an exhaustive reading. In the course of investigating degree relatives we will find answers as to how and under what conditions the two readings hold.

\subsection{The structure of the paper}

The paper is organised as follows. The next section focuses on properties that distinguish degree relatives from ordinary restrictive relatives. Section 3 looks at there-insertion and pseudogapping. When relativised into, these form syntactic contexts that create degree relatives. Section 4 looks at the Carlson/Heim/Grosu $\&$ Landman degree analysis of degree relatives. Section 5 outlines a general picture of semantic interpretation. Section 6 introduces a dynamically quantified modal logic with exhaustification. Section 7 uses this logic to account for the there-insertion and degree relative data. Section 8 looks back at ordinary restrictives. Section 9 looks at free relatives, comparatives, equatives and non-restrictive relatives. Section 10 is the conclusion. 


\section{What's special about degree relatives?}

The most tell-tale property of degree relatives is that they exclude determiners that are not suggestive of an exhaustive reading. So while definites and universals are OK in (6a), (7a) and (8a), indefinites and quantifiers like most and many are out. This is in contrast with the ordinary restrictives (6b), (7b) and (8b), that take the full range of determiners.

(6) a. John has stolen (everything, *something, $\{$ the, $*$ a $\}$ watch $\}$ there was in Mary's bag.

b. John has stolen \{everything, something, \{the, a\} watch $\}$ that was in Mary's bag.

(7) a. They outlined $\{$ the, *some, *four, *most, *many $\}$ differences there are in their various positions.

b. They outlined \{the, some, four, most, many\} differences that are in their various positions.

(8) a. Marv put $\left\{\right.$ everything, $\left\{\right.$ *some $^{*}$ four $\}$ thing(s) $\}$ he could in his pocket.

b. Marv put $\{$ everything, $\{$ some, four $\}$ thing(s) $\}$ he liked in his pocket.

Moreover, degree relatives only receive an exhaustive interpretation. As was noted in Section 1.1, truth conditions for exhaustive and restrictive readings frequently coincide. For example, the two readings coincide in (6a) and (7a). However, they do not for (8a). If given a restrictive reading, (8a) would translate as (9), using $M_{1}$ for Marv could put _ in his pocket and $M_{2}$ for Marv put _ in his pocket.

$$
\forall x\left(M_{1}(x) \rightarrow M_{2}(x)\right)
$$

Suppose the domain of quantification is restricted to the objects in Marv's bedroom. (9) says that if an object in the bedroom can be put in Marv's pocket, it was in fact put there at some time. For example, (9) is false if a pencil that is on the bed is never put in Marv's pocket. But this would not falsify (8a). For (8a) to be true, Marv must only have crammed his pocket full. Significantly, rather than being a statement about each object in the room that can be put in Marv's pocket, (8a) is a statement about Marv putting a certain quantity of things (the largest) in his pocket.

A second property is that degree relatives do not allow the $w h$-relativisers who or which, as shown by (10a), (11a) and (12a). They do however allow that or the empty relativiser. In contrast, the restrictive relatives (10b), (11b) and (12b) allow the full range of relativisers.

(10) a. The men $\{*$ who, that, $\varnothing\}$ there were on this island are dead by now.

b. The men $\{$ who, that, $\varnothing\}$ Crusoe sailed with are dead by now.

(11) a. The light $\left\{{ }^{*}\right.$ which, that, $\left.\varnothing\right\}$ there is in this picture is unlikely to disturb anyone.

b. The light $\{$ which, that, $\varnothing\}$ is/shown in this picture is unlikely to disturb anyone. 
(12) a. Marv put everything $\left\{{ }^{*}\right.$ which, that, $\left.\varnothing\right\}$ he could in his pocket.

b. Marv put everything $\{$ which, that, $\varnothing$ \} he liked in his pocket.

A third property is that degree relatives cannot stack (e.g., (13a) and (13b)), while relatives that take a restrictive reading can (e.g., (13c)). Relatives are stacked when two or more are associated with the same head in a non-conjoined manner.

(13) a. The sailor there was on the boat (*there had been on the island) died.

b. The sailor put everything that he could (*that he should) in his pocket.

c. The sailor who was on the boat (who had been on the island) died.

Finally, Grosu (1994) notes that while the coordinate restrictive relatives in (14a) may be construed as purporting to identify a single set of boys all of whom both sang and danced, the coordinate degree relatives in (14b), do not purport to identify the same set of people.

a. The boys who sang and who danced ...

b. The people that there were at Bill's party and that there had been at Mary's party ...

\section{When do we get degree relatives?}

As Carlson (1977) noticed, degree relatives occur whenever gaps in certain syntactic constructions are relativised into. There be —, as in (6a), (7a), (10a), (11a), (13a) and (14b), creates degree relatives, as will the pseudogapping contexts of (8a), (12a) and (13b). This section focuses on the related properties of these two constructions.

\subsection{There-insertion contexts}

There-insertion contexts have the form in (15). The post-verbal DP is called the "associate".

(15) There verb DP (XP).

(16) shows that the verb has to agree with its associate. Presumably, while there satisfies the EPP (T's need for a specifier), it fails to satisfy the requirement that $T$ check off its number features (and possibly others) (see e.g., Chomsky 1995). Consequently, the features must move to $\mathrm{T}$ from somewhere inside $\mathrm{TP}$, with the associate providing the necessary features.

a. There $\left[\mathrm{F}_{\mathrm{i}}\right.$-is/*are $]\left[\frac{\mathrm{F}}{1} \mathrm{a}\right.$ man $]$ at the door.
b. There $\left[\mathrm{F}_{\mathrm{i}}\right.$-are/*is] [ $\mathrm{F}_{1}^{1}$ some men] at the door.

With data like (17), Milsark (1977), Heim (1987) and others, note that the associate must be an indefinite. 
There is $\left\{\right.$ a man, $*$ the man, $*$ everyone, ${ }^{*} \mathrm{Jim},{ }^{*}$ he/him $\}$ at the door.

Data from bare plurals supports this observation. While (18a) allows both generic ('Books generally are out of stock') and existential ('Some books are out of stock') readings, (18b) has only the existential reading.

(18) a. Books are out of stock.

b. There are books out of stock.

That the associate has to be an indefinite, also rules out the possibility of the associate being a trace. This predicts the impossibility of a reading for (19) where the associate someone outscopes thinks (see e.g., Williams 1984),

(19) John thinks that there is someone in the house.

and predicts that indefinites extracted from the associate position will necessarily undergo scope reconstruction (see e.g., Cresti 1995). This latter prediction is borme out in (20).

(20) How many people do you need there to be at the meeting?

a. *For what $n$ : there are $n$ people $x$ such that you need $x$ to be at the meeting.

b. For what $n$ : you need there to be $n$ people at the meeting.

Similarly, it must be that the degree relatives of (6a), (7a), (10a), (11a), and (14b) have (by LF) indefinite heads in their vacant associate positions.

\subsection{Pseudogapping}

Pseudogapping elides just a verb and typically requires a DP remnant (see e.g., Lasnik 1995):

(21) a. I rolled up a newspaper, and Lynn did a magazine.

b. *Rona sounded annoyed, and Sue did frustrated.

In addition to conjoined sentences like (21a), pseudogapping is found productively in comparatives (e.g., (22)). To be interpreted, comparatives need a thanclause internal head. In a subcomparative like (22), this internal head is realised overtly. Crucially, this licences pseudogapping by providing the DP remnant.

(22) Jane writes more books than Barbara does articles.

Fiengo \& May (1994) use comparatives to show that pseudogapping has a locality effect in contexts in which antecedent-contained deletion (ACD) does not. Thus, (23a), in which the elided verb is understood as writes, contrasts with (23b), in which the elided material would be thought that Jane writes:

(23) a. Mary thought that Jane writes more books than Barbara does articles.

b. *Mary thought that Jane writes more books than Barbara did articles.

Since ACD is found productively in ordinary restrictive relatives, this gives a useful diagnostic for distinguishing pseudogapping from ACD. As (24) shows, 
degree relatives pattern as having pseudogapping, so they must have a (non-overt) DP remnant as well.

(24) a. Mary thinks that Jane stole \{everything, *four paintings Barbara did from Sothebys.

b. *Mary thinks that Jane stole everything Barbara does from Sothebys. (cf. the ACD: Mary thinks that Jane stole everything Barbara does.)

That the DP remnant occurs non-overtly suggests that it is an existential quantifier, since-in anticipation of the semantics introduced in Section 6-this is the simplest form a DP can take. In particular, a non-overt DP will not have restriction material of its own.

\section{So why the degrees?}

Carlson (1977), Heim (1987) and Grosu \& Landman (1998) defend a view of degree relatives that sees them formed by:

(a) the movement to [spec, CP] of a null degree operator,

(b) the deletion in situ, under identity of sense with material within the external head, of the remainder of the nominal in the "gap" position of the relative clause,

(c) the application of a maximalisation operation, which has the effect of restricting the designatum of the $\mathrm{CP}$ to the maximal degree or sum of individuals that falls within the range of abstraction, and

(d) the triggering of a constraint that says the $\mathrm{CP}$ can only combine with determiners that preserve its meaning - and in particular, MAX's output.

For example, this gives the degree relative of (6a) the structure in (25).

(25)

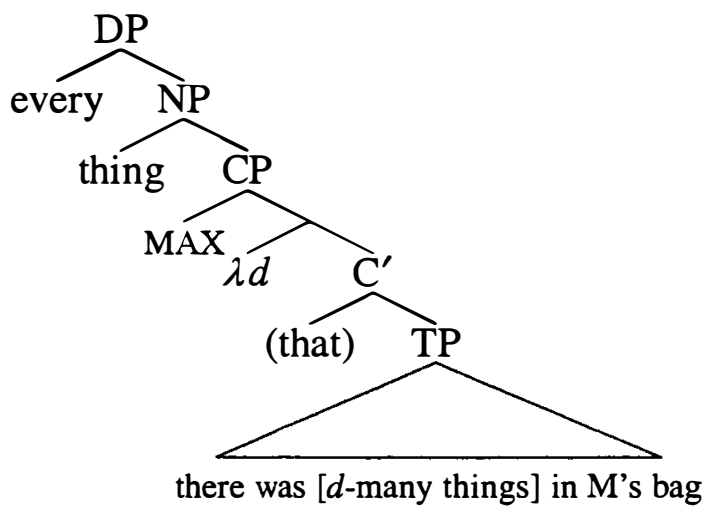

This analysis accounts for the range of data in Section 2 as well as offering an explanation for why there-insertion and pseudogapping contexts create degree relatives. However, it requires stipulation that is limited to the analysis of degree relatives, excluding the possibility of a unified semantics for all relatives. It also has empirical difficulties, since MAX is limited to returning unique maximum values. For example, a paraphrase of $(26)(=(4))$, needs to return a unique minimum value. 
Rullmann (1995) observes that it is possible to get a minimum reading from a maximality operation by inverting the relevant ordering relation. But to work, this requires a non ad-hoc way of choosing the ordering relation's direction. Suppose a way is found, MAX still creates difficulties, since it is limited to retuming unique values. For example, this gives problems in the paraphrase of $(27)(=(8 \mathrm{a}))$.

Marv put everything he could in his pocket.

To paraphrase (27), we first need to decide upon an ordering relation to use. Suppose we settle on $\supset$, giving (28).

Marv put $\operatorname{MAX}_{\supset}(\lambda x$. Marv could put $x$ in his pocket $)$ in his pocket.

The problem with (28) is that it requires that Marv put in his pocket the unique collection of things that make up all he could put in his pocket. But this is not an entailment of (27). As an alternative, we might use (29).

Marv put $\max _{>}(\lambda y . \exists x($ Marv could put $x$ into his pocket $\wedge$ $\operatorname{VOLUME}(x)=y))$ in his pocket.

This will give a representation that avoids requiring uniqueness by saying that Marv put in his pocket something(s) with maximum volume. But to work (29) has relied on the addition of extra restriction material, namely $\operatorname{VOLUME}(x)=y$, that is not part of (27). ${ }^{6}$

As a solution to these problems, I will abandon using degrees and use exhaustification instead. Exhaustification can be used to paraphrase (27) as (30), using $M_{1}$ for Marv could put _ in his pocket and $M_{2}$ for Marv put _ in his pocket.

$$
\exists x\left(M_{1}(x) \wedge \neg \exists y\left(x \neq y \wedge M_{1}(y) \wedge \square\left(M_{1}(y) \rightarrow M_{1}(x)\right)\right) \wedge M_{2}(x)\right) .
$$

This says that Marv put in his pocket something which he could put in his pocket, where there was nothing stronger that he could put in his pocket. Because of the meaning of $M_{1}$, stronger equates to something like 'larger in volume'. Significantly, this does not require that Marv put in his pocket something with the unique property of involving an amount for which it was not possible to further cram his pocket. Rather he put in his pocket something from a set of potentially many things (as determined by the model) meeting this criterion.

Having established that degree relatives are more successfully analysed with exhaustification, there is a new question to answer: how does natural language employ this strategy?

\section{The interpretation process}

With a dynamic view of interpretation (e.g., Groenendijk \& Stokhof 1991, Harel et al. 2000), words can be viewed as actions that read an input, perform a simple transformation, and write an output. To make this idea more concrete, consider how it might apply to (31). 
Assume the first sentence translates as an existential quantifier, which has the role of an independent atomic formula. This can be taken to add a new object to its input (provided there is someone in the domain, that is; otherwise the sentence is false). The resulting output is the kind of input needed to interpret the second sentence. This is especially the case if he is associated with the newly introduced object. This can be accomplished by translating the pronoun as the index $\boldsymbol{n}+1$, which takes as its denotation the contents of the context location $c_{n+1}$. The second sentence then tests for whether the contents of $c_{n+1}$ is walking in the park. If the test succeeds, its input is passed on as output. This process might be pictured as in (32), using < for "takes as input" and > for "gives as output":

$$
\exists<c_{1} \ldots c_{n}>c_{1} \ldots c_{n} c_{n+1} . W(n+1)<c_{1} \ldots c_{n}\left|c_{n+1}>c_{1} \ldots c_{n}\right| c_{n+1} .
$$

In addition to the existential quantifier and simple predicate tests, a dynamic logic has available a third type of operation: the ability to quantify over inputs and outputs. In what follows, I will call operators that do this control operators. The propositional connectives are typical examples of control operators.

\section{Smart DQMLE}

In this section, I introduce the semantics used in the remainder of the paper. This is a smart dynamic quantified modal logic with exhaustification (henceforth, $D Q M L E)$. The logic is based on van Eijck's (2001) Incremental Dynamics system (see also Dekker's (1994) system of Predicate Logic with Anaphora).

The logic is 'smart' because it builds controls on the context into the syntax of its formulas. The logic does not use variables, but instead uses term indices that link up with locations from the input context, which is always a sequence of objects from the domain. Each formula is a pair $(n, \phi)$, where $n$ gives the size of the input context, and $\phi$ is made from the following vocabulary:

DEFINITION 6.1. The primitive non-logical vocabulary of $D Q M L E$ is a set $R C$ of relation constants: $P_{1}^{n}, P_{2}^{n}, \ldots(n \geq 1)$. I will use capitalised relation names like $P, Q, \ldots$ as typical members of $R C$.

In addition to the non-logical vocabulary, $D Q M L E$ uses the following logical vocabulary:

[1] The set $\mathbb{N}^{+}$of positive natural numbers;

[2] The control operators ; , $\rightarrow, \neg, \square, T$ and $E$, the identity sign = , the quantifier $\exists$, and T.

In clause 3 of the definition below, if $X$ is a non-empty finite set of indices from $\mathbb{N}^{+}, \sup (X)$ gives the maximum, 0 otherwise (the supremum function). 
DEFINITION 6.2. The set of $D Q M L E$ formulas, $\mathrm{L}$, is the smallest set containing:

[1] $(n, \mathrm{~T})$ whenever $n \in \mathbb{N}$;

[2] $\quad(n, \exists ; \phi)$ provided $(n+1, \phi) \in \mathrm{L}$;

[3] $\left(n, P^{m}\left(v_{1}, \ldots, v_{m}\right) ; \phi\right)$ provided $P^{m} \in R C, v_{1}, \ldots, v_{m} \in \mathbb{N}^{+}$, $\sup \left(\left\{v_{1}, \ldots, v_{m}\right\}\right) \geq n$ and $(n, \phi) \in \mathrm{L}$;

[4] $\left(n, v_{1}=v_{2} ; \phi\right) \in \mathrm{L}$ provided $v_{1}, v_{2} \in \mathbb{N}^{+}, \max \left(v_{1}, v_{2}\right) \geq n$ and $(n, \phi) \in \mathrm{L}$

[5] $(n,(\phi \rightarrow \psi) ; \chi) \in \mathrm{L}$ provided $(n, \phi) \in \mathrm{L},(n+e(\phi), \psi) \in \mathrm{L}$ and $(n, \chi) \in \mathrm{L}$

[6] $(n,(\neg \phi) ; \psi) \in \mathrm{L}$ provided $(n, \phi) \in \mathrm{L}$ and $(n, \psi) \in \mathrm{L}$;

[7] $\left(n,\left(T_{\nu} \phi\right) ; \psi\right) \in \mathrm{L}$ provided $v \in \mathbb{N}^{+},(n, \phi) \in \mathrm{L}$ and $(n+e(\phi), \psi) \in \mathrm{L}$;

[8] $(n,(\square \phi) ; \psi) \in \mathrm{L}$ provided $(n, \phi) \in \mathrm{L}$ and $(n, \psi) \in \mathrm{L}$;

[9] $\left(n,\left(E_{X} \phi\right) ; \psi\right) \in \mathrm{L}$ provided $X \subseteq \mathbb{N}^{+},(n, \phi) \in \mathrm{L}$ and $(n+e(\phi), \psi) \in \mathrm{L}$.

The presentation of syntax is not yet complete, since control operators that are externally and/or internally dynamic need to know the 'existential depth' of a formula. Notation for this is $e(\phi)$. Intuitively, the existential depth of $(n, \phi)$ calculates the number of positions by which an input sequence has grown after the semantic processing of $\phi$. For example, the existential depth of $(n, \exists ; \mathrm{T})$ is 1 , for any $n$. If $(n, \phi) \in \mathrm{L}$, the existential depth of $\phi$ is given by:

\section{DEFINITION 6.3. Existential depth:}

$$
\begin{aligned}
e(T) & :=0 \\
e(\exists ; \phi) & :=1+e(\phi) \\
e\left(P\left(v_{1}, \ldots, v_{n}\right) ; \phi\right) & :=e(\phi) \\
e\left(v_{1}=v_{2} ; \phi\right) & :=e(\phi) \\
e((\phi \rightarrow \psi) ; \chi) & :=e(\chi) \\
e((\neg \phi) ; \psi) & :=e(\psi) \\
e\left(\left(T_{v} \phi\right) ; \psi\right) & :=e(\phi)+e(\psi) \\
e((\square \phi) ; \psi) & :=e(\psi) \\
e\left(\left(E_{X} \phi\right) ; \psi\right) & :=e(\phi)+e(\psi)
\end{aligned}
$$

This completes the presentation of syntax. Every formula has the form $\left(n, \phi_{1} ; \ldots ; \phi_{k} ; \mathrm{T}\right)$, with $k>0$. If $k>0$, I will write $\left(n, \phi_{1} ; \ldots ; \phi_{k} ; \mathrm{T}\right)$ as $\left(n, \phi_{1} ; \ldots ; \phi_{k}\right)$. I will omit unnecessary parentheses whenever possible. For example, I will write the formula $(3, \neg(R(2,3)))$ as $3, \neg R(2,3)$, etc. Also, I will usually write $\exists ; \phi$ as $\exists \phi$. Thus L contains such formulas as in (33). Notably, (34) is not a well-formed formula because $e(\exists \rightarrow P(1))=0$ and $e(\exists)=1$, so that 2 , the argument of $Q$, is not less than or equal to the size of the context, which is $0+0+1$ at the point of its interpretation.
a. $0, \exists \exists$.
b. $4, P(3,2,4)$.
c. $2, \exists Q(1,3)$.
d. $0,(\exists \rightarrow P(1)) ; \exists Q(1)$. 


$$
\text { * } 0,(\exists \rightarrow P(1)) ; \exists Q(2) \text {. }
$$

Satisfaction for $D Q M L E$ is defined relative to a quantified modal logic model with constant domain (see e.g., Fitting \& Mendelsohn 1998). This takes the form: $\llbracket n, \phi \rrbracket^{M, w}<\sigma>\tau$. $(n, \phi)$ is an L-formula. $M$ is a model. $w$ is a world from the set of worlds in $M$. A world assigns to each predicate symbol with arity $n$ an $n$-arity relation on $D$ (the domain of $M$ ). $\sigma$ is an input (a.k.a. the anaphoric context). $\tau$ is an output. Inputs and outputs $\{\sigma, \tau, \theta, \zeta, \ldots\}$ are finite sequences of elements from $D$. The set of all finite sequences of elements of $D$ is denoted $D^{*}$.

Upon meeting an existential quantifier, an input $\sigma$ gets extended with a single element $o$ from $D$. Notation for this is $\sigma^{\wedge} o$. The concatenation of two finite sequences $\sigma, \tau \in D^{*}$, in that order, is written as $\sigma^{\wedge} \tau$. The length of a finite sequence $\sigma$ is denoted $l(\sigma)$. The set of all finite sequences of elements of $D$ of length $n$ is denoted $D^{n}$. The unique element of $D^{*}$ of length 0 is called the empty sequence and is denoted $\varepsilon$. The $n$-th element of $\sigma$ is denoted $\sigma[n] . \operatorname{diffsim}_{X}(\zeta, \theta)$ is used to say that finite sequences $\zeta, \theta$ differ and that they differ at most in their $n$-th elements, where $n \in X$.

DEFINITION 6.4. A model $M$ for the non-logical vocabulary $R C$ is any quadruple $\langle W, D, R, V\rangle$ satisf ying the following conditions:

[1] $\quad W$ is a non-empty set of worlds;

[2] $D$ is a non-empty domain of objects;

[3] $R$ is a binary accessibility relation on $W$, i.e., $R \subseteq W \times W$;

[4] $V$ is a valuation function where if $P^{n} \in R C$, $V\left(P^{n}\right) \in\left\{g \mid g: W \rightarrow \wp\left(D^{n}\right)\right\}$.

DEFINITION 6.5. The denotation of term $v$ with respect to finite sequence $\sigma$ is defined as follows:

$$
\llbracket v \rrbracket_{\sigma}:=\left\{\begin{array}{l}
\sigma[v] \text { if } v \leq l(\sigma), \\
\text { undefined otherwise }
\end{array}\right.
$$

Note that the proviso $l(\sigma)=n$ in the satisfaction clauses guarantees that the term functions $\llbracket v_{i} \rrbracket_{\sigma}$ in clauses 3 and 4 are well defined.

DEFINITION 6.6. The input output relations $\sigma, \tau$ satisfy $_{M}$ L-formula $(n, \phi)$ with respect to world $w$ (in symbols: $\llbracket n, \phi \rrbracket^{M, w}<\sigma>\tau$ ) as follows:

[1] $\llbracket n, T \rrbracket^{M, w}<\sigma>\tau$ iff $\sigma=\tau$;

[2] $\llbracket n, \exists ; \phi \rrbracket^{M, w}<\sigma>\tau$ iff $l(\sigma)=n \& \exists o \in D\left(\llbracket n+1, \phi \rrbracket^{M, w}<\sigma^{\wedge} o>\tau\right)$;

[3] $\llbracket n, P\left(v_{1}, \ldots, v_{m}\right) ; \phi \rrbracket^{M, w}<\sigma>\tau$ iff $l(\sigma)=n \&$

$\left\langle\llbracket v_{1} \rrbracket_{\sigma}, \ldots, \llbracket v_{m} \rrbracket_{\sigma}\right\rangle \in[V(P)](w) \& \llbracket n, \phi \rrbracket^{M, w}<\sigma>\tau ;$

[4] $\llbracket n, v_{1}=v_{2} ; \phi \rrbracket^{M, w}<\sigma>\tau$ iff $l(\sigma)=n \& \llbracket v_{1} \rrbracket_{\sigma}=\llbracket v_{2} \rrbracket_{\sigma} \& \llbracket n, \phi \rrbracket^{M, w}$ $<\sigma>\tau$;

[5] $\llbracket n,(\phi \rightarrow \psi) ; \chi \rrbracket^{M, w}<\sigma>\tau$ iff $l(\sigma)=n \& \forall \theta \in D^{*}\left(\llbracket n, \phi \rrbracket^{M, w}<\sigma>\theta\right.$ $\left.\Rightarrow \exists \zeta \in D^{*}\left(\llbracket n+e(\phi), \psi \rrbracket^{M, w}<\theta>\zeta\right)\right) \& \llbracket n, \chi \rrbracket^{M, w}<\sigma>\tau$;

[6] $\llbracket n,(\neg \phi) ; \psi \rrbracket^{M, w}<\sigma>\tau$ iff $l(\sigma)=n \& \neg \exists \theta \in D^{*}\left(\llbracket n, \phi \rrbracket^{M, w}<\sigma>\theta\right) \&$ $\llbracket n, \psi \rrbracket^{M, w}<\sigma>\tau$; 
[7] $\llbracket n,\left(T_{\nu} \phi\right) ; \psi \rrbracket^{M, w}<\sigma>\tau$ iff $l(\sigma)=n \& \exists \theta \in D^{e(\phi)}(1 \leq v-n \leq l(\theta) \&$ $\left.\llbracket n, \phi \rrbracket^{M, w}<\sigma>\sigma^{\wedge} \theta \& \llbracket n+e(\phi), \psi \rrbracket^{M, w}<\sigma^{\wedge} \theta>\tau\right)$;

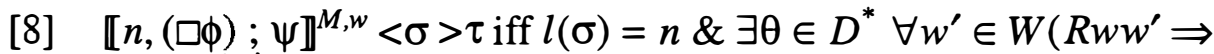
$\left.\llbracket n, \phi \rrbracket^{M, w^{\prime}}<\sigma>\theta\right) \& \llbracket n, \psi \rrbracket^{M, w}<\sigma>\tau$;

[9] $\llbracket n,\left(E_{X} \phi\right) ; \psi \rrbracket^{M, w}<\sigma>\tau$ iff $l(\sigma)=n \& \exists \theta \in D^{*}\left(\llbracket n, \phi \rrbracket^{M, w}<\sigma>\theta \&\right.$ $\neg \exists \zeta \in D^{*}\left(\operatorname{diffsim}_{X}(\zeta, \theta) \& \llbracket n, \phi \rrbracket^{M, w}<\sigma>\zeta \& \forall w^{\prime} \in W\left(R w w^{\prime} \&\right.\right.$ $\left.\left.\left.\llbracket n, \phi \rrbracket^{M, w^{\prime}}<\sigma>\zeta \Rightarrow \llbracket n, \phi \rrbracket^{M, w^{\prime}}<\sigma>\theta\right)\right) \& \llbracket n+e(\phi), \psi \rrbracket^{M, w}<\theta>\tau\right)$.

DEFINITION 6.7. Controlled differences and similarities of finite sequences $\sigma$ and $\tau$ :

$\operatorname{diffsim}_{X}(\sigma, \tau)$ iff $l(\sigma)=l(\tau)=n \& \exists v \in X(\sigma[v] \neq \tau[v]) \&$

$\forall m \in \mathbb{N}^{+}((m \leq n \& \neg \exists v \in X(m=v)) \Rightarrow \sigma[m]=\tau[m])$.

DEFINITION 6.8. Truth:

$(n, \phi)$ is true with respect to model $M$, world $w$, and input sequence $\sigma$ iff $\exists \tau \in D^{*}\left(\llbracket n, \phi \rrbracket^{M, w}<\sigma>\tau\right)$.

\section{The semantics applied}

\subsection{Simple "there be _" sentences}

To capture the data in (17)-(20), I will take the presentational context there be to be the control operator $T_{v}$, with feature movement from the associate setting the value of $v$. In scoping over a formula $\phi, T_{v}$ works by introducing a new context sequence $\theta$ of length $e(\phi)$. It appends $\theta$ to its input $\sigma$ and requires this to be an output of the $\phi$-transition when $\sigma$ is given as input. It moreover requires that $v-l(\sigma) \geq 1$ and $v-l(\sigma) \leq e(\phi)$. That is, that the sequence location $v$ is not part of the input context $\sigma$ and that it is present in the context once the $\phi$-transition completes.

This analysis gives, for example, (35a) the L-formula (35b/c/d). (35b/c/d) is interpretable because $l(\varepsilon)=0, v=1$ and $e(\exists ; M(1) ; D(1))=1$, so that $1-0 \geq 1$ and $1-0 \leq 1$.

(35) a. There is a man at the door.

b. 0 , There $\left[\mathrm{F}_{1}\right.$-is] [ $\mathrm{F}_{\mathrm{T}} \mathrm{a}$ man] at the door $<\varepsilon>$ peter ${ }^{7}$

c. $0, T_{1}$ ([F-a man] at the door) $<\varepsilon>$ peter .

d. $0, T_{1}(\exists ; M(1) ; D(1))<\varepsilon>$ peter .

It also gives (36a) the L-formula (36b/c),
a. ${ }^{*}$ There is $\{$ Peter, he/him $\}$ at the door. ${ }^{8}$
b. 1 , There $\left[\mathrm{F}_{1}\right.$-is] $\left[\mathrm{F}_{\mathrm{T}} 1\right]$ at the door $<$ peter $>*$.
c. $1, T_{1}(D(1))<$ peter $>*$.

and (37a) the L-formula (37b/c). 
(37) a. *There is everyone at the door.

b. 0 , There $\left[\mathrm{F}_{1}\right.$-is] $\left[\mathrm{F}_{1}\right.$-everyone] at the door $<\varepsilon>*$.

c. $0, T_{1}(\exists \rightarrow D(1))<\varepsilon>*$.

(36b/c) fails to retum an output because $l$ (peter) $=1$ and $v=1$, so that $1-1 \geq 1$, and (37b/c) fails to return an output because $l(\varepsilon)=0, v=1$ and $e(\exists \rightarrow D(1))=0$, so that $1-0 \leq 0$. The same reasoning captures the data in (18)-(20).

\subsection{Now for the relatives}

I will suppose that the control operator $E_{X}$ is the topmost part of every CP-projection, and that wh-movement's raison d'être is to raise phrases to [spec, $\mathrm{CP}$ ] to place their indices in $X$. Since $D Q M L E$ can only introduce values with an existential quantifier, relatives will have the following possible schemata:

$$
\begin{array}{ll}
\text { a. } & n, \ldots E_{\{n+1\}}(\ldots \exists \ldots) \ldots \\
\text { b. } & n, \ldots \exists \ldots E_{\{n+1\}}(\ldots) \ldots
\end{array}
$$

I will call the existential quantifiers in (38) semantic heads (henceforth, s-heads). An s-head forms the instruction to add to the context the object that the relative clause is about. In (38a) the s-head is internal to the relative clause, while in (38b) it is outside the relative clause.

$E_{X} \phi$ works by ensuring that there is no output $\zeta$ that is different from $\theta$ with respect to an $n$-th element, where $n \in X$, and stronger than $\theta$. An output $\zeta$ will only be stronger than $\theta$ if it is a different output and every accessible world that supports $\phi$ with input $\sigma$ and output $\zeta$ also supports $\phi$ with input $\sigma$ and output $\theta$. If no output $\zeta$ can be found, then $\theta$ must be exhaustive with respect to the contents of $X$.

Taking $\sigma$ to be the input and $\theta$ to be the output of a transition, note that the only source for variation between $\sigma$ and $\theta$ will be from extensions to $\sigma$ brought about by occurrences of existential quantifiers. Also, note that $E_{X}$ 's presence in a formula will have no noticeable effect if all indices in $X$ happen to be greater than $l(\theta)$. Consequently, $E_{X}$ will only have an effect in situations where $\sigma$ and $\theta$ differ and there are indices $n$ in $X$ such that $l(\sigma)<n \leq l(\theta)$.

Hence, of the two possible schemata for relatives, (38a) and (38b), it is only in (38a) that the exhaustification operator has an effect on the interpretation, requiring the s-head to take on an exhaustive value with respect to the contents of the formula over which $E$ scopes. Importantly, despite the fact that $E$ has no observable effect in (38b), its application is not vacuous in the sense that it has nothing to quantify over. Rather it carries out the same semantic computation as in (38a), quantifying over the outputs of the transition over which it scopes and all accessible worlds.

\subsection{Now for the relatives that relativise into "there be _"}

Of the possible structures for a relative relativising into there be $\rightarrow,(39 a)$ is the only structure licensed by $T$. Here, $T$ has as its associate the relative's s-head. As a consequence, the s-head finds itself under the influence of $E$, forcing it to take an exhaustive interpretation. 

a. $n, \ldots E_{\{n+1\}}\left(\ldots T_{n+1}(\ldots \exists \ldots) \ldots\right) \ldots$
b. $\# n, \ldots E_{\{n+1\}}\left(\ldots \exists \ldots T_{n+1}(\ldots) \ldots\right) \ldots$
c. $\# n, \ldots \exists \ldots E_{\{n+1\}}\left(\ldots T_{n+1}(\ldots) \ldots\right) \ldots$

This buys us a solution to the puzzle of why degree relatives are limited to exhaustive readings. It gives, for example, (40) the interpretable structure (40b/c).

(40) a. John has stolen everything there was _ in Mary's bag.

b. $n$, John has stolen $\left[\mathrm{CP} E_{\{n+1\}} \exists\right.$ there $\left[\mathrm{F}_{\mathrm{n}+1}\right.$-was] $\left[\mathrm{F}_{\mathrm{n}+\mathrm{T}} \exists\right]$ in Mary's bag]. wh-movement followed by reconstruction

c. $n, E_{\{n+1\}} T_{n+1} \exists B(n+1) ; J(n+1)$.

(40b/c) shares the truth conditions of (41a), which, given the usual interpretation of $B$ (was in Mary's bag), shares the truth conditions of (41b).
a. $\exists x(B(x) \wedge \neg \exists y(y \neq x \wedge B(y) \wedge \square(B(y) \rightarrow B(x))) \wedge J(x))$.
b. $\forall x(B(x) \rightarrow J(x))$.

\subsection{What about the relative clause external material?}

If there is an internal s-head then this will fix the denotation of the relative clause, so that any external material takes on the form of supporting in kind the fixed denotation. I will suppose that such material is used as a processing aid, but that it does not contribute to the semantics. Since $E$ makes the denotation of the internal s-head exhaustive, the only external determiners acceptable will be those that continue to guarantee exhaustivity, e.g., definites and universals, ruling out indefinites, cardinals, most, many, etc.

\subsection{The associate as a degree relative}

It is possible to have a degree relative as the associate of a there-insertion context. On the face of it, this creates a problem. While the there-insertion context, of which the degree relative is the associate, needs an indefinite, the degree relative itself, as shown by (42), needs a non-indefinite exhaustive determiner.

(42) There will be \{everyone, the people, *four people $\}$ that there should be at the party.

As (43b/c) shows, conflict is averted under the current analysis, with the degree relative's internal s-head taking over the semantic contribution of every, to bring about the change in context that both occurrences of there are primed to check for.

(43) a. There will be everyone that there should be at the party.

b. $n$, There will [ $\mathrm{F}_{n+1}$-be] [evene [CP $E_{\{n+1\}} \exists$ that there should [ $\mathrm{F}_{n+\Gamma}$ be] $\left[\mathrm{F}_{n+1} \exists\right]$ at the party]] at the party.

c. $n, T_{n+1}\left(E_{\{n+1\}} T_{n+1} \exists P_{1}(n+1) ; P_{2}(n+1)\right)$.

$(43 \mathrm{~b} / \mathrm{c})$ shares the truth conditions of (44a), which, given the usual interpretation of $P_{1}$ (there should be _ at the party), shares the truth conditions of (44b). 

a. $\exists x\left(P_{1}(x) \wedge \neg \exists y\left(y \neq x \wedge P_{1}(y) \wedge \square\left(P_{1}(y) \rightarrow P_{1}(x)\right)\right) \wedge P_{2}(x)\right)$.
b. $\forall x\left(P_{1}(x) \rightarrow P_{2}(x)\right)$.

\subsection{Restrictives relativising into pseudogapping}

A non-overt internal DP remnant saves (45a). And since it is non-overt, it must be an existential quantifier, which is the simplest form a DP can take. This gives (45a) the interpretable structure $(45 \mathrm{~b} / \mathrm{c})$, with the internal s-head taking over the semantic contribution of every.
a. Marv put everything he could _ in his pocket.
b. $n$, Marv put [ his pocket.
c. $n, E_{\{n+1\}} \exists x M_{1}(n+1) ; M_{2}(n+1)$.

Notably, while $(45 \mathrm{~b} / \mathrm{c})$ is truth conditionally equivalent to $(46)(=(30))$, it is not, given the usual interpretation of $M_{1}$ (Marv could put _ in his pocket), equivalent to $(47)(=(9))$.

$$
\begin{aligned}
& \exists x\left(M_{1}(x) \wedge \neg \exists y\left(x \neq y \wedge M_{1}(y) \wedge \square\left(M_{1}(y) \rightarrow M_{1}(x)\right)\right) \wedge M_{2}(x)\right) . \\
& \forall x\left(M_{1}(x) \rightarrow M_{2}(x)\right)
\end{aligned}
$$

\subsection{The restriction on relativisers explained}

Who and which in ordinary restrictives (e.g., (10b), (11b) and (12b)) have to be abstraction operators. Suppose they can only be abstraction operators. Their exclusion from (10a) and (11a) follows, since they would take up the structural slot needed by the s-head that licences there be _. Additionally, to function, abstraction operators must not reconstruct, which is why pseudogapping's DP remnant requirement is not satisfied in (12a).

\subsection{The inability to stack explained}

Relatives in a stack join together via set-intersection to act as a restriction on a single s-head (see e.g., Partee 1973). This is only possible if the s-head is outside the stacked relatives. Having (the potential to have) external s-heads, ordinary restrictives can stack (e.g., (13c)). Since relatives that relativise into there be and pseudogapping contexts must have internal s-heads, they cannot stack (e.g., $(13 a, b))$.

\subsection{Coordinated degree relative behaviour explained}

The coordinate restrictive relatives in (14a) can take on the LF structure (48), which purports to identify a single set of boys all of whom both sang and danced.

$$
\begin{aligned}
& n, \text { The } \lambda x .[\lambda y .[y \text { boys }](x) ; \\
& \lambda y .\left[\lambda z .\left[{ }_{\mathrm{CP}} E_{\{z\}} z \text { sang }(y) ; \lambda z .\left[{ }_{\mathrm{CP}} E_{\{z\}} z \text { danced }\right](y)\right](x)\right] \ldots
\end{aligned}
$$

In contrast, such a structure is not available for the coordinate degree relatives in (14b). Instead, the degree relatives must each contain an s-head to satisfy their 
respective $T$ operators. This gives (14b) the LF structure (49). This is the reason why the degree relatives of $(14 b)$ do not purport to identify the same set of people.

$n$, The people $\left[{ }_{\mathrm{CP}} E_{\{n+1\}}\left[\exists\right.\right.$ people] that $T_{n+1}\left[\mathrm{~F}_{n+1}[\exists\right.$ people $\left.]\right]$ at Bill's party] and [ ${ }_{\mathrm{CP}} E_{\{n+2\}}$ people] that $T_{n+2}\left[\mathrm{~F}_{n+2}[\exists\right.$ people $\cdots$

\section{Ordinary restrictives}

As was noted in Section 1.1, ordinary restrictives are in principle ambiguous, having their expected restrictive reading and an exhaustive reading. For example, consider (50) $(=(1))$.

(50) Peter ate everything that would fit in his pocket.

Here, the relative clause has nothing to force an exhaustive reading (it does not relativise into a there be - or pseudogapping context), but then it has nothing to exclude such a reading either (it uses everything and that). So we should expect both readings. In particular, (50) can be given either of the LF structures in (51):

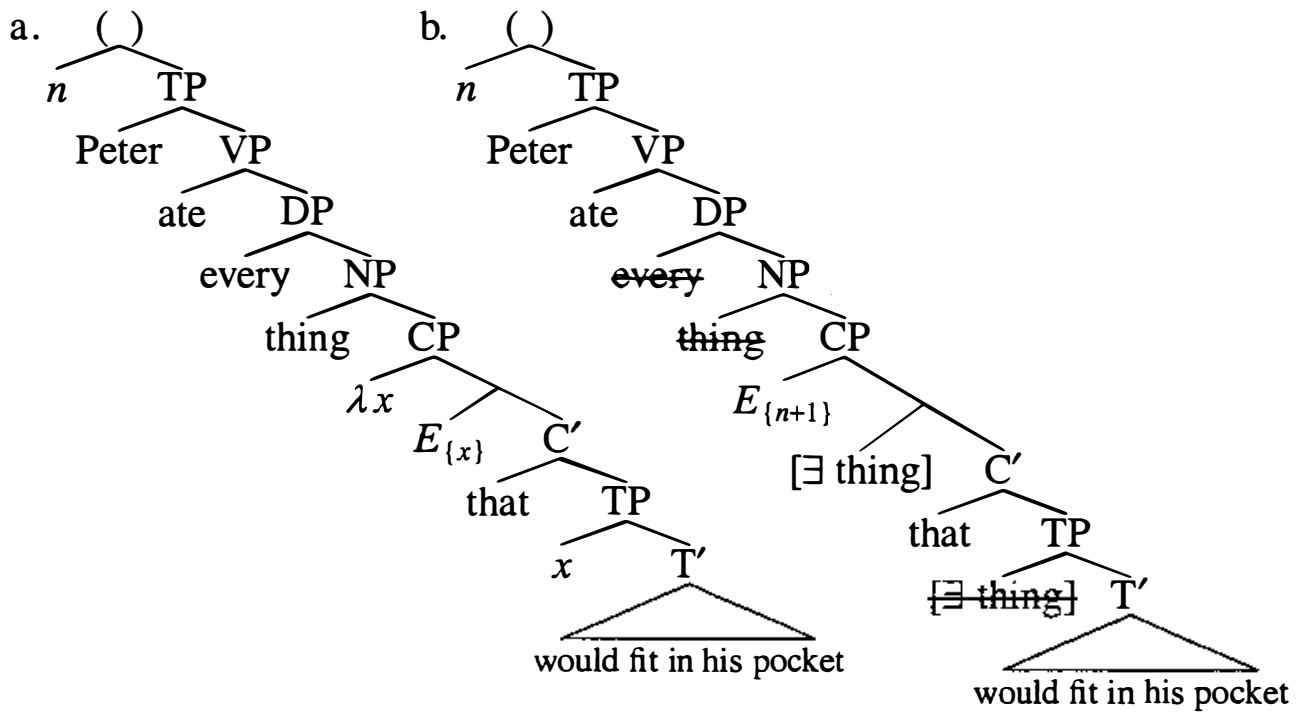

The LF (51a) forms the interpretable (52). This is truth conditionally equivalent to (53), which is the restrictive reading of (1).

$$
\begin{aligned}
& n, \exists \rightarrow\left(E_{\{n+1\}} P(n+1) \rightarrow A(n+1)\right) . \\
& \forall x(P(x) \rightarrow A(x)) .
\end{aligned}
$$

The LF (51b) forms the interpretable (54). This is truth conditionally equivalent to (55), which is the exhaustive reading of (1).

$$
\begin{aligned}
& n, E_{\{n+1\}} \exists P(n+1) ; A(n+1) . \\
& \exists x(P(x) \wedge \neg \exists y(x \neq y \wedge P(y) \wedge \square(P(y) \rightarrow P(x))) \wedge A(x)) .
\end{aligned}
$$


Notably, for the restrictive reading, the s-head occurs outside the relative clause as part of every, while it is generated non-overtly inside the relative clause for the exhaustive reading. ${ }^{9}$ This accounts for why (in principle) ordinary relatives have the means to create restrictive and exhaustive readings. It follows that a degree relative is an ordinary relative which has had its ability to create a restrictive reading blocked (for example, by the independent properties of syntactic constructions like there-insertion and pseudogapping). Likewise, unambiguously restrictive relatives (relatives with non-exhaustive determiners, $w h$-relativisers or relatives that form part of a stack) are ordinary relatives that have had their ability to create exhaustive readings blocked.

\section{Other relative clause-like constructions}

What there be _ and pseudogapping have in common is that they bring about relative clause internal s-heads. Other constructions with (observable) internal sheads include free relatives, comparatives and equatives. This section looks at these constructions and at non-restrictive relatives, in the context of the current analysis.

\subsection{Free relatives}

Free relatives can relativise into the gap of a there be _ context (e.g., (56)), they can save a pseudogapping context (e.g., (57)), they cannot stack (e.g., (58)), and when coordinated as in (59), they fail to carry the implication of being about the same thing(s). Hence, their behaviour parallels degree relative behaviour.

(56) John has stolen what there was _ in Mary's bag.

(57) Marv put what he could _ in his pocket.

(58) *Whoever was on the boat (*whoever was on the island) died.

(59) What(ever) John bought and what(ever) Mary sold ...

This is strong evidence in support of free relatives having internal s-heads (see e.g., Groos \& van Riemsdijk 1981), from which it follows, for example, that (57) gets the interpretable structure $(60 \mathrm{a} / \mathrm{b})$, which is correctly predicted to receive the distinctive exhaustive reading $(61)(=(30))$.

(60) a. $n$, Marv put [CP $E_{\{n+1\}}$ he could put what in his pocket] in his pocket.

b. $n, E_{\{n+1\}} \exists x M_{1}(n+1) ; M_{2}(n+1)$.

$$
\exists x\left(M_{1}(x) \wedge \neg \exists y\left(x \neq y \wedge M_{1}(y) \wedge \square\left(M_{1}(y) \rightarrow M_{1}(x)\right)\right) \wedge M_{2}(x)\right) .
$$

\subsection{Comparatives and equatives}

Comparatives and equatives can relativise into there be _ contexts (e.g., (62) and (63)), they can relativise into pseudogapping contexts (e.g., (64) $(=(22)))$, and they cannot stack (e.g., (65)). Hence, their behaviour parallels degree relative 
behaviour.

(62) John has stolen more money than there was _ in Mary's bag.

(63) I had never seen such a sparkle as there was _ in your eyes.

(64) Jane writes more books than Barbara does _ articles.

(65) More sailors than were on the boat (*than were on the island) died.

Comparatives need a than-clause internal s-head that returns an exhaustive value. Similarly, equatives need an as-clause internal s-head that returns an exhaustive value. These facts explain the degree relative behaviour, and follow from having $E$ always introduced by the CP-projection. For example, this gives (66a) the interpretable structure (66b/c), using $P$ for Peter can jump _ high and $M$ for Mary can jump _ high.
a. Peter can jump higher than Mary can jump.
b. $n$, Peter can jump higher than [CP $E_{\{n+2\}}[\exists$ high] Mary can jump [قhigh].
c. $n, \exists P(n+1) ; E_{\{n+2\}} \exists M(n+2) ; n+1>n+2$.

In $(66 \mathrm{~b} / \mathrm{c})$, the internal existential quantifier falls under the influence of $E$, making it equivalent to (67).

$$
\exists x(P(x) \wedge \exists y(M(y) \wedge \neg \exists z(z \neq y \wedge M(z) \wedge \square(M(z) \rightarrow M(y))) \wedge x>y) .
$$

Given the usual interpretation of $M$ (Mary can jump — high), this makes (66a) equivalent to (68) (cf. von Stechow 1984).

$$
\exists x(P(x) \wedge x>\max (\lambda y . \exists M(y))) .
$$

\subsection{Non-restrictives}

Non-restrictive relatives require $w h$-relativisers that act essentially as anaphoric pronouns. This gives, for example, (69a) the L-formula (69b/c), which leaves $E$ without influence. This makes $(69 \mathrm{~b} / \mathrm{c})$ equivalent to (70). Notably, if $E$ had influence, then it would have entailed that you only wanted to meet Peter, which is not an entailment of (69a).

(69) a. Peter, who you wanted to meet, has an inspiring face.

b. 1, 1 has an inspiring face <peter $>$ peter. $1,\left[{ }_{\mathrm{CP}} E_{\{1\}} 1\right.$ you wanted to meet 1 ] < peter $>$ peter.

c. $1, F(1)<$ peter $>$ peter. $1, E_{\{1\}} M(1)<$ peter $>$ peter.

$$
A(\text { peter }) \wedge M(\text { peter }) \text {. }
$$

As expected, since non-restrictives have external s-heads, they cannot relativise into the associate position of a there-insertion context, as (71) shows, but can stack, as (72) shows. 
(71) *A boy, who there was with his Mum, didn't wish to enter. (cf. A boy, who was with his Mum, didn't wish to enter.)

(72) Peter, who you wanted to meet, who will be at the Hotel Europa this weekend, has an inspiring face.

\section{Conclusion}

I have given a mechanism of exhaustification that feeds off the observation that it is the location of the s-head that causes degree relative behaviour. This accounts for the full range of degree relative facts without using degrees, and gives a general syntax/semantics applicable to relatives of all kinds.

\section{Endnotes}

* This work was supported by the AHRB under grant reference 98/3159, which is gratefully acknowledged. I thank David Adger, Steve Harlow, Julia Hockenmaier, George Tsoulas, Henk Zeevat and the participants of SALT XI for their useful comments and suggestions. Errors, of course, are my sole responsibility.

1. The exhaustive reading is often called the maximal reading in the literature (see e.g., Grosu \& Landman 1998). But I argue in what follows that maximality is too restrictive a notion.

2. I will assume plural domains here and throughout the paper.

3. More because if $R(x)$, it necessarily holds that $R(y)$ for $x \supset y$, where $R$ is Peter read__ books.

4. The minimum because if $M_{1}(x)$, it necessarily holds that $M_{1}(y)$ for $x<y$, where $M_{1}$ is Peter can live on _ money.

5. The maximum because if $M_{2}(x)$, it necessarily holds that $M_{2}(y)$ for $x>y$, where $M_{1}$ is Peter can spend_money.

6. Also there is a problem of a more technical nature: $\mathrm{MAX}_{>}$closes off the binding domain of the quantifiers within its scope, preventing $\exists x$ from binding the main clause predicate Marv put _ in his pocket.

7. The output here is one of potentially many, with the condition that the newly introduced value is a man and at the door.

8. Anaphoric reference is used here to simulate the constant peter.

9. For the exhaustive reading, in addition to the s-head, restriction material that matches the descriptive content of the $\mathrm{CP}$ external material needs to be generated non-overtly inside the relative clause. However, the control structure of the CP external material has to be lost-namely every's contribution: $(\ldots \rightarrow(\ldots \rightarrow \ldots))$.

\section{References}

Butler, Alastair. 2001. 'Sentences with Exhaustification', in H. Bunt, I. van der Sluis and E. Thijsse (eds.), Proceedings of the Fourth International Workshop on Computational Semantics IWCS-4, Computational Linguistics and 
AI Group, Tilburg, pp. 21-34.

Carlson, Greg. 1977. 'Amount Relatives', Language 53, 520-542.

Chomsky, Noam. 1995. The Minimalist Program, MIT Press, Cambridge.

Cresti, Diana. 1995. 'Extraction and Reconstruction', Natural Language Semantics 3, 79-122.

Dekker, Paul. 1994. Predicate logic with anaphora (seven inch version), in L. Santelmann and M. Harvey (eds.), Proceedings of SALT IV, DMLL Publications, Cornell University, 79-95.

van Eijck, Jan. 2001. 'Incremental dynamics', Journal of Logic, Language and Information 10, 319-351.

Fitting, Melvin, and Richard L. Mendelsohn. 1999. 'First-Order Modal Logic', Kluwer Academic Publishers, Dordrecht.

Groenendijk, Jeroen and Stokhof, Martin. 1991. 'Dynamic predicate logic', Linguistics and Philosophy 14, 39-100.

Groos, Anneke and Henk van Riemsdijk. 1981. 'Matching effects in free relatives: a parameter of core grammar', in A. Belletti et al. (eds.), Theory of Markedness in Generative Grammar, Scuola Normale Superiore, Pisa, pp. 171-216.

Grosu, Alexander. 1994. Three Studies in Locality and Case, Routledge, London.

Grosu, Alexander, and Fred Landman. 1998. 'Strange Relatives of the Third Kind', Natural Language Semantics 6, 125-170.

Fiengo, Robert, and Robert May. 1994. Indices and Identity, MIT Press, Cambridge.

Harel, David, Dexter Kozen and Jerzy Tiuryn. 2000. Dynamic Logic, MIT Press, Cambridge.

Heim, Irene. 1987. 'Where Does the Definiteness Restriction Apply? Evidence from the Definiteness of Variables', in E. Reuland and A. ter Meulen (eds.), The Representation of (In)definiteness, MIT Press, Cambridge, $21-42$.

Lasnik, Howard. 1995. 'A Note on Pseudogapping', in R. Pensalfini and H. Ura (eds.) Papers on Minimalist Syntax, MIT Working Papers in Linguistics 27, 143-163.

Milsark, G. 1977. 'Toward an explanation of certain peculiarities of the existential construction in English', Linguistic Analysis 3, 1-29.

Partee, Barbara. 1973. 'Some Transformational Extensions of Montague Grammar', Journal of Philosophical Logic 2, 509-534.

Rullmann, Hotze. 1995. Maximality in the Semantics of Wh-constructions, Ph. D. Thesis, University of Massachusetts at Amherst.

von Stechow, Amim. 1984. 'Comparing Semantic Theories of Comparison', Journal of Semantics 3, 1-77.

Williams, Edwin. 1984. 'There-insertion', Linguistic Inquiry 15, 131-153.

Zeevat, Henk. 1994. 'Applying an Exhaustivity Operator in Update Semantics', in H. Kamp (ed.), Ellipsis, Tense, and Questions, DYANA-deliverable, ILLC, Amsterdam, 233-269. 